\title{
A prospective survey of the causes of non-traumatic spastic paraparesis and tetraparesis in $\mathbf{5 8 5}$ patients
}

\author{
A Peter Moore and Lance D Blumhardt* \\ The Walton Centre for Neurology and Neurosurgery, Walton Hospital, Rice Lane, Liverpool L9 1AE, U.K.
}

\begin{abstract}
Objective: To ascertain the relative frequencies of the causes of non-traumatic paraparesis and tetraparesis in adults. Design: Survey of patients enrolled prospectively over a 3 year period between 1986 and 1989 and review of their case notes 1 year after enrollment ceased (mean duration of follow up 30 months). Setting: Regional neurosciences centre in the UK serving over three million people in Merseyside and North Wales. Patients: Experienced clinicians from the centre saw most patients in the region with non-traumatic spastic paraparesis or tetraparesis. Primary investigation of patients was by myelography, for which patients were admitted to the centre. 585 consecutive patients with spastic paraparesis or tetraparesis were identified by daily screening of all 2104 patients undergoing myelography or radiculography during the 3 year period, ie selection by the intention to investigate them for myelopathy. Exclusions: age under 15 years, previous myelography for myelopathy. Interventions: None. Main outcome measures: Numbers and proportions of patients with each condition or category of disease. Results: Commonest diagnoses were cervical spondylotic myelopathy $(23.6 \%)$, extrinsic neoplastic or developmental tumour $(16.4 \%)$, multiple sclerosis $(9.1 \%$ rising to $17.8 \%$ after MRI of a selected group), and motor neurone disease $(4.1 \%)$. Diagnosis was uncertain in $27.4 \%$, falling to $18.6 \%$ after MRI. Conclusions: This survey shows the pattern of diseases producing non-traumatic myelopathy in the Mersey Region and in North Wales. Changing patterns of referral, investigation in peripheral hospitals and by non neurologically trained practitioners, and increasing use of outpatient MRI for primary investigation may make comparable surveys impossible in the future.
\end{abstract}

Keywords: myelography; spinal cord diseases; epidemiology; magnetic resonance imaging

\section{Introduction}

Previous surveys of spastic paraparesis or tetraparesis have been retrospective $\mathrm{s}^{3,4,6,12,13}$ or have concentrated on selected groups such as patients with acute myelopathy ${ }^{4}$ or chronic progressive myelopathy. $6,9,12$ Some surveys have included a high proportion of paediatric (including spina bifida) or trauma cases. ${ }^{2} \mathrm{We}$ have carried out a prospective study of a large group of patients recruited at the time of their first investigation for non-traumatic spastic paraparesis or tetraparesis. The study was approved by the institution's ethical committee.

\section{Materials and methods}

The Mersey Regional Neurosciences Centre is the only neurological service for over 3 million people in Merseyside and North Wales. During the period of

Correspondence: AP Moore

*Current address: Division of Clinical Neurology, Faculty of Medicine University Hospital, Queen's Medical Centre, Nottingham NG7 2UH, UK this study it admitted most of the patients in the region requiring investigation for spastic paraparesis or tetraparesis, except patients with acute traumatic spinal lesions who were routinely dealt with elsewhere.

In the 3 year period betwen 1986 and 1989 myelography was the routine primary investigation for possible myelopathy, with selected patients undergoing subsequent CT or MRI of the spine. Each day we examined the day-book in the radiology department and prospectively identified all patients undergoing myelography or radiculography over this period. After perusal of X-ray request forms, review of the notes, and if necessary examination of the patient, all patients were identified who were aged 15 years or more, had signs of a spastic paraparesis or tetraparesis and were undergoing their first myelogram for that condition. We did not personally examine patients in whom it was clear that a consultant neurologist or neurosurgeon had made a clinical diagnosis of spastic paraparesis or tetraparesis.

For this selected group we collected information on clinical features. We recorded the myelogram findings 
and the diagnosis after further investigation or surgery. One year after the survey was completed we reviewed each patient's notes and revised the final diagnosis if necessary. We did not attempt to influence patient management.

\section{Results}

The pattern of admissions did not change between 1986 and $1989 .^{8} 2104$ patients underwent myelography or radiculography during this period (Table 1), 585 (306 men, 279 women) of whom had spastic paraparesis or tetraparesis under investigation for the first time.

The primary anatomical indication for myelography was spastic paraparesis in 450 and spastic tetraparesis in 135 . On clinical grounds 366 patients appeared to have an isolated myelopathy, with additional involvement of cranial structures (hemisphere, cerebellum, brain stem or cranial nerves) in 111 , or of peripheral root or nerve in 108. In 166 patients the clinician suggested the likely aetiology before myelography (Table 2), although in many other cases the probable aetiology was implied but not specified.

\section{Results of myelography}

The results of myelography appear in Table 3a. Table $3 b$ shows the conclusions drawn on the basis of the clinical picture and results of myelography and basic CSF tests (excluding immunology). The diagnosis remained uncertain in 246/585 (42\%) patients ie the largest single group, which included many patients who were eventually found to have multiple sclerosis (MS).

\section{Final diagnoses}

Table 4 shows the diagnoses reached after further imaging studies including MRI in a few cases, CT and angiography, neurophysiology including electromyo-

\section{Table 1}

a: Patient screening and selection

Myelogram subjects screened

Patients with myelopathy undergoing first myelogram

Male

Female

b: Indications for investigation in patients included in study

(a) Primary anatomical indication

Spastic paraparesis

Spastic tetraparesis

Total

(b) Other regions involved

None - isolated myelopathy

Cranial: hemisphere, brain stem,

366

cerebellum, cranial nerves

Root or peripheral nerve

Total graphy (EMG), nerve conduction studies and evoked potentials, blood and CSF tests, biopsy or operation. It includes information obtained from the review of case notes a year after case ascertainment was completed.

Uncertain diagnosis There were $160(27.4 \%)$ patients in whom the diagnosis remained uncertain before MRI. It is likely that some in this group have MS. Sixty-nine of the 160 cases were under 50 years old and had an isolated, unexplained and clinically definite myelopathy with no other complicating systemic disease. They all had MRI and were followed up. They are the subject

Table 2

\begin{tabular}{lr}
\hline Likely aetiology specified prior to myelogram & \\
None & 419 \\
Metastasis from known tumour elsewhere & 43 \\
Congenital: developmental anomalies apparent & 31 \\
Skeletal abnormalities & 23 \\
Multiple sclerosis & 18 \\
Trauma & 14 \\
Motor neurone disease & 10 \\
Pathology added to pre-existent multiple sclerosis & 8 \\
Familial or inherited & 5 \\
Other & 14 \\
Total & 585 \\
\hline
\end{tabular}

Table 3 Result of myelogram

\begin{tabular}{lrc}
\hline (a) anatomical & $\mathrm{n}$ & $(\%)$ \\
& 277 & 48 \\
Compressed cord - definite & 225 & 39 \\
Normal & 23 & 3.9 \\
Compressed cord - equivocal & 22 & 3.8 \\
Cord enlargement & 8 & 1.3 \\
Lumbar root compression & 6 & 1 \\
Cervical root compression & 6 & 1 \\
Atrophy of cord & 3 & 0.5 \\
Arachnoiditis & 15 & 2.5 \\
Other & 585 & \\
Total & & \\
(b) Diagnosis on basis of clinical, myelographic and & \\
basic CSF findings & & \\
& $\mathrm{n}$ & $(\%)$ \\
Uncertain & 246 & 42 \\
Cervical spondylotic myelopathy & 141 & 24 \\
Extrinsic tumour & 102 & 17 \\
Multiple sclerosis & 21 & 3.6 \\
Congenital spinal lesion & 12 & 2.1 \\
Syringomyelia & 11 & 2.0 \\
Intrinsic tumour & 11 & 2.0 \\
Rheumatoid cervical degeneration & 8 & 1.3 \\
Arteriovenous malformation & 5 & 0.9 \\
Thoracic disc & 4 & 0.7 \\
Arachnoiditis & 3 & 0.5 \\
Trauma & 3 & 0.5 \\
Lumbar disc & 2 & 0.3 \\
Other & 16 & 2.7 \\
Total & 585 & \\
\hline
\end{tabular}


of a separate report ${ }^{1}$ : 51/69 (74\%) had MRI scans consistent with MS (ie clinically probable MS ${ }^{10}$ ). After an average of 22 months follow up 12/69 (17\%) had developed clinically definite MS. If these diagnoses made by MRI are included in the present survey the number with an uncertain diagnosis falls to $109 / 585$ $(18.6 \%)$, and the number with MS rises to $104 / 585$ $(17.8 \%)$. We would probably have detected more cases of MS if we had been able to investigate more of the patients in the 'uncertain' group using MRI. However, the possibility of other pathologies including vascular disease might have made interpretation difficult.

Primary skeletal disease $(n=168 / 585,28.7 \%)$ This was the largest group with a clear diagnosis, and included $138(23.8 \%)$ patients with cervical spondylotic myelopathy. Eighty-five $(62 \%)$ of these promptly went on to operation. Others may have had surgery at a later date. Four patients had thoracic disc protrusions requiring immediate surgery.

There were $11(1.9 \%)$ patients who had cord deformity related to a congenital or developmental spinal disease such as Arnold-Chiari (3) or KlippelFeil (1) anomalies, tethered cord with thickened filum terminale (4), achondroplasia (1), lumbar dysraphism (1) or kyphoscoliosis of uncertain aetiology (1). Many of the 14 patients with syringomyelia also had congenital spinal or foramen magnum anomalies.

Eight $(1.4 \%)$ patients had cervical cord compression with lesions due to known rheumatoid arthritis; three cases had atlanto-axial instability without evidence of rheumatoid disease. Other acquired skeletal causes of myelopathy included Paget's disease (2), hypopho-

Table 4 Diagnosis with additional tests (not MRI) and follow up

\begin{tabular}{lrr}
\hline & $\mathrm{n}$ & $(\%)$ \\
Uncertain & 160 & 27.4 \\
(Still uncertain after MRI of selected group) & 109 & 18.6 \\
Cervical spondylotic myelopathy & 138 & 23.6 \\
Exrinsic neoplastic or developmental tumour & 96 & 16.4 \\
Multiple sclerosis (MS) & 53 & 9.1 \\
(MS after MRI of selected group) & 104 & 17.8 \\
Motor neurone disease & 24 & 4.1 \\
Intrinsic tumour, including AVM & 15 & 2.5 \\
Syringomyelia & 14 & 2.4 \\
Congenital spine & 11 & 1.9 \\
Other vascular disease (brain 5, cord 5) & 10 & 1.7 \\
Parenchymal immune disease other than MS & 8 & 1.4 \\
Spinal abscess & 8 & 1.4 \\
Rheumatoid cervical spine & 8 & 1.4 \\
Non-metastatic tumour effects & 6 & 1.0 \\
Primary neurological degenerative disease & 6 & 1.0 \\
Familial cord disease & 6 & 1.0 \\
Infectious disease (not abscess) & 4 & 0.7 \\
Thoracic disc & 4 & 0.7 \\
Trauma & 4 & 0.7 \\
Arachnoiditis & 2 & 0.3 \\
Other & 8 & 1.4 \\
Total & 585 & \\
\hline
\end{tabular}

sphataemic rickets (1), and ankylosing spondylitis (1). Neoplasms A breakdown of the tumour type in the $96 /$ $585(16.5 \%)$ patients with an extrinsic compressive tumour and the $15 / 585(2.6 \%)$ patients with an intrinsic tumour is given in Tables 5 and 6 .

Previously known malignancy There were 41/585 (7\%) patients with a history or clinical evidence of neoplasia prior to myelography. In only two of these was an independent non-cancerous pathology (cervical spondylotic disease) identified, with no evidence of tumour recurrence or iatrogenic disease. In 24 of the 41, spread or recurrence of neoplasia was confirmed as the cause of cord compression, though two of these were assumed diagnoses as no definitive histology was available. A second primary tumour appeared in three patients. Non-metastatic tumour related myelopathy was diagnosed or strongly suspected in six. Iatrogenic radiation necrosis was eventually blamed in three patients and three others had arachnoiditis related to previous encephalograms. One of these had a possible recurrence of cranial ependymoma which made the cause of the spastic paraparesis uncertain.

Table 5 Histology or organ of origin of extrinsic compressive tumours

Nervous system: meningioma (10), $(\%)$ neurofibroma (4), schwannoma (1), neurilemmoma (1), neuroblastoma (1), ependymoma (2) 20

Haemopoietic: myeloma (6), non-Hodgkins lymphoma (5), lymphoma (3), Hodgkins (1), plasmacytoma (1)

Carcinoma (site unidentified)

Cysts: arachnoid (2), dermoid (3), mesenchymal (1)

Mesenchyme: chondrosarcoma (2), osteochondroma (2), osteogenic sarcoma (1), osteoclastoma (1), liposarcoma (1)

Prostate

Lung

Breast

Melanoma

Ovary

Renal

Thyroid

Testis

Uncertain

Total

$\begin{array}{ll}16 & 17\end{array}$

$13 \quad 14$

$\begin{array}{ll}7 & 7\end{array}$

Table 6 Histology of intrinsic cord tumours

$\begin{array}{lr}\text { No histology (AVM) } & 4 \\ \text { Astrocytoma } & 3 \\ \text { Ependymoma } & 3 \\ \text { Haemangioblastoma } & 2 \\ \text { No history (other) } & 2 \\ \text { Benign intramedullary cyst } & 1 \\ \text { Total } & 14\end{array}$


Parenchymal immune disease $(n=61 / 585, \quad 10.4 \%)$ There were 53 patients in whom a diagnosis of clinically probable or clinically definite MS was made. ${ }^{10} \mathrm{~A}$ further eight had transverse myelitis of uncertain aetiology (3), encephalomyelitis clearly related to recent viral illness or immunisation (3), SLE (1) or sarcoidosis (1).

Infections $(n=14 / 585,2.4 \%)$ Eight patients had a spinal abscess: staphylococcus aureus was isolated in five, E. coli in one, one had a tuberculoma and one was sterile. There were five patients with positive syphilis serology. One had classical tabes dorsalis, another had infarction of the cord presumed to be caused by meningovascular disease, and a third had CSF changes suggesting active syphilis. It was not clear whether syphilis contributed to the myelopathy in the other two, one of whom may have had yaws. One patient had Creutzfeldt-Jacob disease. The 69 (white, European) patients with pure myelopathy and normal myelograms who were being followed long-term were all negative for HTLV-1. ${ }^{5}$ Patients were not tested for HIV.

Neurodegenerative and familial There were 36/585 $(6.2 \%)$ patients in this group. They had motor neurone disease (24), spinocerebellar degeneration (5), hereditary spastic paraparesis (2), spinal muscular atrophy (2), multi-system atrophy (1), adrenomyeloleukodystrophy (1), and possible corticobasal degeneration (1).

Vascular disease $(n=10,1.7 \%)$ Infarction of the cord related to atherosclerotic disease was diagnosed in five patients. In five patients a clinical syndrome mimicking cord disease was eventually attributed to multi-infarct or brainstem cerebrovascular disease.

Metabolic disease There were four patients with mild or moderately severe B12 deficiency. They were all treated with B12 supplements, but it was not clear whether the B12 deficiency was relevant to the myelopathy. One had significant coexisting cervical spondylotic cord compression. In all four the final diagnosis was registered as uncertain.

\section{Age profiles}

Age profiles are given in Figure 1 for patients with cervical spondylotic myelopathy, compressive neoplasms, MS and motor neurone disease. The proportion with MS peaked in the decade 40-49. The proportions with cervical spondylotic myelopathy, neoplasms and motor neurone disease continued to rise until the decade $60-69$. The subsequent apparent fall in incidence of these three disorders is probably an artefact of our screening technique in that increasingly elderly patients are less likely to be investigated by myelography. Comparison of the groups with MS and with pure, non-compressive myelopathy (not shown) showed a bulge in the early decades and a relative decline after age 49 for the latter group.

\section{Discussion}

Although this was a hospital based survey, the results are probably representative of significant non-traumatic myelopathy in our community. Case ascertainment was prospective and based on the clinically defined picture of myelopathy as determined by experienced clinicians. Inclusion in the study was on the basis of intention to investigate for myelopathy. Apart from cases obviously related to trauma which were seen in spinal injury units, significant myelopathy arising in our area would almost always result in referral to one of our clinicians. There could have been overspill to adjacent neurological services, but this would probably affect all causes of myelopathy equally. Patients were routinely investigated in our unit rather than at the referring hospital. As myelography was used as the first choice investigation at the time, all patients who were investigated were detected and entered into the study.

Nevertheless, some patients with myelopathy will not have been included in this study ie patients with mild sensory symptoms suggestive of myelopathy but not severe enough to warrant investigation, and others in whom it was not considered appropriate to undertake myelography because of severe systemic illness or advanced cancer. Not all patients with myelopathy due to known MS or motor neurone disease will have been subjected to myelography.

It should be noted that of the 2104 patients screened, only those with signs consistent with spastic paraparesis or tetraparesis were entered into the study. There were undoubtedly other patients who could have had spinal cord disease without signs of upper motor neurone involvement - for example, patients with pure root or lower motor neurone signs or symptoms, ataxia, pure bladder dysfunction or pain with no other signs. These cases were not entered.

This was not a survey of isolated myelopathy. Patients with additional neurological features such as cranial nerve or spinal root disease were included. Such features rarely preclude spinal cord disease, and we aimed to generate an overall picture of the causes of non-traumatic myelopathy.

The modern first choice investigation of myelopathy is MRI, and myelography is much less frequently used. It is possible that this shift in practice will reveal differing patterns of myelopathy, but only $18.6 \%$ of our patients were left undiagnosed in this survey, and either possible pathology or multiple pathologies were uncovered in some of these.

\section{Reasons for an uncertain final diagnosis}

Multiple sclerosis It is likely that many of the patients in whom a final diagnosis could not be 

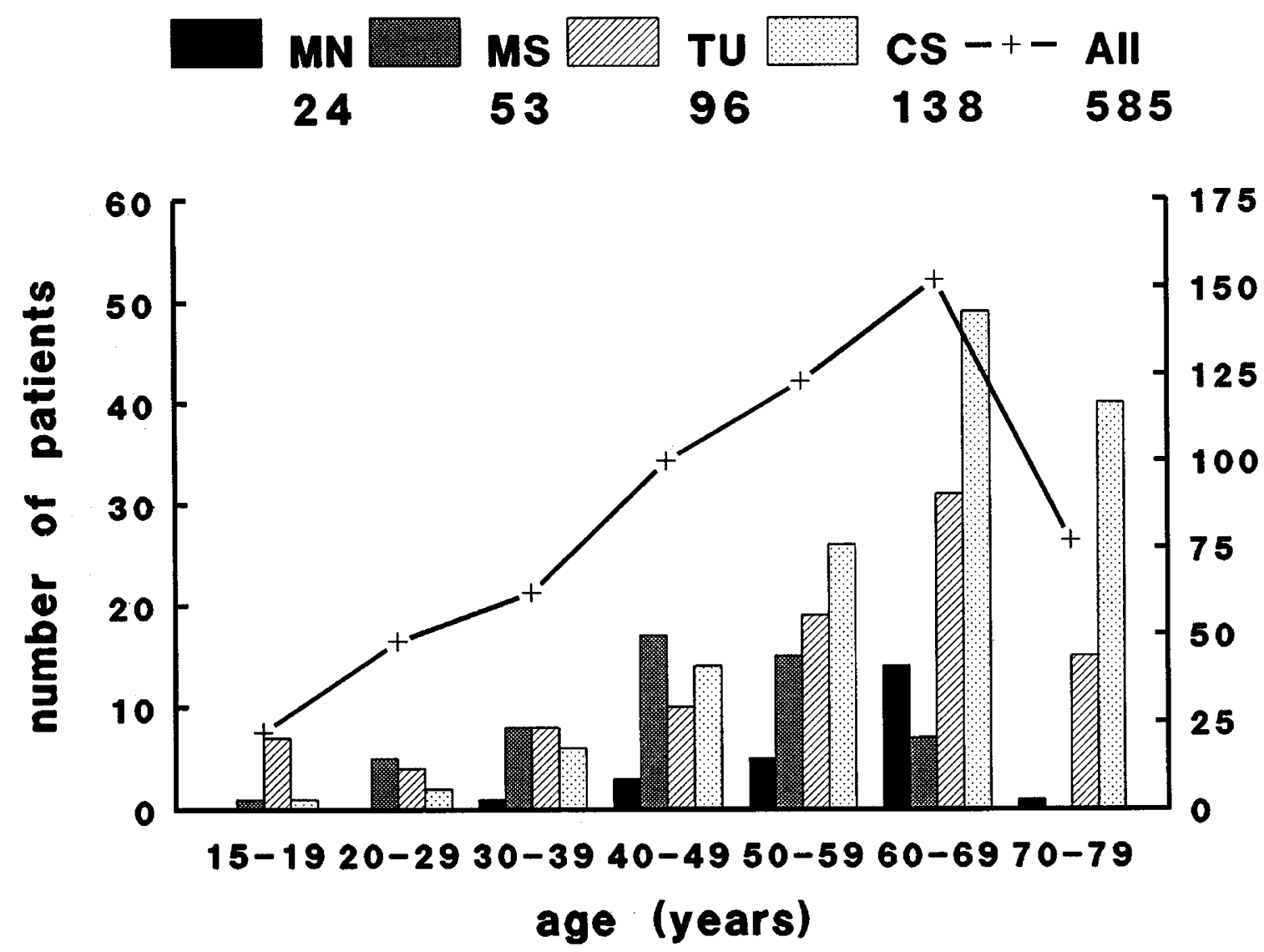

Figure 1 Histogram showing age profiles for patients with motor neurone disease (MN), multiple sclerosis (MS), compressive tumour (TU) and cervical spondylotic myelopathy (CS). Left axis shows scale for individual conditions, right axis shows scale for line graph of total number of patients in each age group, including all other diagnoses. The lower numbers of patients with MND, TUM and CSM in the decade 70-79 years probably reflect both a smaller population and the method of case selection, with a reluctance to pursue myelography in elderly patients. Numbers in the age group $80-89$ years were too small to be worth including

confirmed will eventually develop MS. In previous studies of myelopathy follow up revealed MS in $3 \%{ }^{4}-75 \%{ }^{7}$ depending on the initial case selection, the extent of the diagnostic screen, and the duration of follow up. It is difficult to compare reports because these factors either varied widely in each study or were not discussed.

Motor neurone disease This diagnosis may also be under-represented at the time of myelography and may only become apparent with time. It is important because a number of the patients diagnosed as having other disorders, particularly cervical spondylotic myelopathy, may have motorneurone disease in addition, or even as the sole contributory pathology (Rowland 1992). ${ }^{11}$ They are likely to fare less well after surgery.

Multiple pathology This category has not been used in previous studies. We encountered diagnostic difficulty in many patients because of multiple potentially relevant pathologies. For instance, one patient had possible radiation myelitis after treatment for ankylosing spondylitis, and was also found to have bilateral subdural haematomas. Some patients with significant cervical spondylotic cord compression had a family history or signs such as pes cavus suggesting an associated familial myelopathy, or showed features of MS. There were numerous other combinations which made an unequivocal single diagnosis impossible, so that in 14 such patients the diagnosis remained uncertain. The clinician felt able to decide which was the dominant pathology in other patients who had more than one possible aetiology.

Insufficient evidence of causation Thirty patients had suggestive but not diagnostic abnormalities; they included the four who were treated for past or present B12 deficiency, and two with a history of syphilis which may have been inadequately treated many years earlier. There were six patients with possible ischaemic cerebrovascular or cord disease, five patients with 
features suggestive of sporadic familial disease and three with congenital or developmental anomalies not sufficiently pronounced to be diagnostic.

Not myelopathy Multi-infarct or brain stem cerebrovascular disease (five patients), lumbar disc disease (1), and polymyositis (1) were occasionally mistaken for myelopathy and the patient subjected to myelography.

\section{Comparison with previous studies}

The only comparable published study is that of Ungar-Sargon, Lovelace and Brust (1980), who carried out a survey of 672 patients admitted to the Columbia Presbyterian Medical Centre over 16 years with spastic paraplegia-paraparesis (SPP) as a prominent finding. ${ }^{13}$ Their study differed from ours in a number of ways. It was retrospective and a majority $(77 \%)$ of the group did not have myelography. Patients were not entered on the basis of an 'intention to investigate', nor was there any information supplied about factors leading to admission. No age ranges were given or limits applied except for patients in whom the diagnosis remained uncertain. Their study group comprised 181 patients with spinal trauma, and 491 others. To allow comparison with our data we have excluded the trauma patients from the following discussion and modified their original figures accordingly.

CT and MRI scanning were not available to UngarSargon and colleagues. ${ }^{13}$ In our study cranial CT may have influenced case selection in that some patients with intracranial causes of SPP may have been diagnosed without myelography. We would not have recruited these patients. However, CT and (in a few patients) MRI after myelography enabled us to make or exclude some diagnoses with greater certainty.

Ungar-Sargon and co-workers assigned diagnoses to 520 patients 'on the basis of history, clinical findings or plain spine radiography without myelography or other further investigations'. ${ }^{13}$ It is not clear how the final diagnoses were achieved in many of these cases, or what degree of certainty was required. $108 / 491(21 \%)$ were diagnosed after investigations which included myelography, spinal angiography, CSF analysis or surgery. In 44/491 $(9 \%)$, all with 'pure' progressive SPP, no diagnosis was reached. This group compares best with the 69/ $585(12 \%)$ of our patients with undiagnosed isolated myelopathy, ${ }^{1}$ although our group included patients with monophasic and relapsing disease. Overall, we could not reach a definite single diagnosis in 160/585 $(27.4 \%)$ patients. If the results of MRI scanning in our selected group of $69 / 585$ are included, the number undiagnosed falls to $109(18.6 \%)$.

A diagnosis of cervical spondylotic myelopathy was made in only $15 / 491(3 \%)$ of the patients studied by Ungar-Sargon et al, ${ }^{13}$ compared to $139 / 585(24 \%)$ of our patients. This striking and significant difference (Chi-square with Yates' correction, $P<0.0001$ ) is difficult to explain unless there were major differences in referral patterns. Marshall $(1955)^{6}$ found in a retrospective survey that only $5 / 87(6 \%)$ of his patients with 'progressive spastic paraplegia' of middle age had cervical spondylosis, but clearly this was a highly selected group. ${ }^{6}$

The proportions of patients with tumours in the two studies were comparable at $14 \%$ and $17 \%$ respectively. The distribution of tumour type was also similar, with $6 / 60(10 \%)$ of the patients with a spinal tumour having an intrinsic neoplasm in the New York study, compared to $14 / 110(13 \%)$ of our patients. Metastatic disease accounted for 35/60 (58\%) and 58/110 (53\%) respectively. In patients with metastatic disease UngarSargon et $\mathrm{al}^{13}$ found $5 / 35(14 \%)$ to be of haemopoietic origin compared to our $16 / 58(28 \%)$. This difference is not significant. The primary sites of origin were otherwise similar in the two series. 15/60 (25\%) of the American tumour cases had extrinsic tumours of nervous system origin compared to $19 / 110(17 \%)$ in our series.

Ungar-Sargon and colleagues classified arteriovenous malformation separately, recording 21/491 (4\%) diagnosed by tortuous vessels on myelography or by spinal angiography, ${ }^{13}$ compared to our figure of $6 / 585$ $(1 \%)$. Other differences include their finding of $10 / 491$ $(2 \%)$ with arachnoiditis (they did not specifically exclude patients with previous or multiple myelograms) compared to $2 / 585(0.3 \%)$ of our patients, and $10 / 491(2 \%)$ with amyotrophic lateral sclerosis (ALS) against our 24/585 (4.1\%) with motor neurone disease.

There were 4/491 patients with Pott's disease or tuberculosis and no mention is made of other organisms causing spinal abscess. In our 8/585 patients with spinal abscess, five were due to staphylococcus aureus and only one related to tuberculosis. Positive syphilis serology was uncommon in both series, 4/491 versus 5/585, and in both series it was not clear whether the syphilis was the primary pathology in all cases.

\section{Changing patterns of disease}

The difference in the design of the two studies makes it difficult to draw conclusions about changing patterns of disease. The larger proportion left undiagnosed in our study may reflect its prospective nature, the shorter period of follow up and differing criteria for diagnosis. The most striking difference was the higher proportion with cervical spondylotic myelopathy in our study $(24 \%$ versus $4 \%)$, although this could be a referral or selection artefact. In future, changing patterns may arise through new diseases such as HIV-related myelopathy.

\section{Future surveys}

The increasing availability of MRI will alter the apparent spectrum of diagnoses in a number of ways. It may help to diagnose conditions such as MS, and to 
reduce the number of patients in whom the diagnosis remains uncertain. However, more sensitive investigations may also increase the proportion of patients in whom dual pathology is detected, thus making a clear diagnosis more difficult. Indirectly, the threshold for investigation is likely to fall, more patients will be dealt with locally by doctors not specifically trained in the neurosciences, and referral patterns will change. These factors suggest that for epidemiological surveys any gain in accuracy of diagnosis using MRI as the primary investigation might be countered by poorer case selection and the loss of the representative overview previously available to regional units. It will not be possible to compensate for this by using diagnosis-related codes from district hospitals because these would permit only retrospective surveys.

\section{Conclusion}

This survey shows the pattern of diseases producing non-traumatic myelopathy in Mersey region and North Wales. Case selection was by intention to investigate for myelopathy. A representative sample and high standard of case selection were ensured because most patients with non-traumatic myelopathy were seen at the regional centre. Myelography was the primary investigation, only undertaken after clinical consultation with a fully trained neurologist or neurosurgeon, and all patients undergoing myelography were clinically screened. It is likely to be the last time that such a study can be undertaken, as MRI is now the imaging technique of choice in this situation. In any future comparisons the method of case selection is probably the most important factor to consider.

\section{Acknowledgements}

This study was supported by MRHA Research Grant 527.

\section{References}

1 Blumhardt LD et al. A prospective study of 'undiagnosed' isolated myelopathy: II. Value of magnetic resonance imaging, evoked potentials and CSF analysis. European Journal of Neurology 1995; 2: 307-315.

2 Buchan AC et al. A preliminary survey of the incidence and aetiology of spinal paralysis. Paraplegia 1972; 10: $23-28$.

3 Hübbe P, Dam AM. Spastic paraplegia of unknown origin. A follow up of 32 patients. Acta Neurol Scand 1973; 49: 536-542.

4 Lipton HL, Teasdall RD. Acute transverse myelopathy in adults. A follow-up study. Archives Neurology 1973; 28: 252-257.

5 Longson M, Blumhardt LD, Moore AP. Negative HTLV-1 serology in 'undiagnosed' myelopathy in North West England and North Wales. J Neurol Neurosurg Psychiatry 1991; 54: $185-$ 186

6 Marshall J. Spastic paraplegia of middle age. A clinicopathological study. Lancet 1955: 643-646.

7 Miller DH et al. Magnetic resonance imaging in isolated noncompressive spinal cord syndromes. Annals Neurology 1987; 22: 714-723.

8 Moore AP, Blumhardt LD. Audit study of a programmed investigation unit. J Neurol Neurosurg Psychiatry 1991; 54: 269 271.

9 Paty DW et al. Chronic progressive myelopathy: investigation with CSF electrophoresis, evoked potentials, and CAT scan. Trans Amer Neurol Assoc 1978; 103: 110 - 112 .

10 Poser CM et al. New diagnostic criteria for multiple sclerosis: guidelines for research protocols. Annals Neurology 1983; 13: $227-231$.

11 Rowland LP. Surgical treatment of cervical spondylotic myelopathy: time for a controlled trial. Neurology 1992; 42: 4-13.

12 Steiner I et al. Chronic progressive myelopathy: its relation to the spinal progressive form of multiple sclerosis. Acta Neurol Scand 1988; 77: $152-157$

13 Ungar-Sargon JY, Lovelace RE, Brust JM. Spastic paraplegiaparaparesis: a reappraisal. Journal of Neurological Science 1980; 46: $1-12$. 